\title{
Supplementary Information Nuclear Magnetic Resonance Measurements and Electronic Structure of Pu(IV) in $\left[(\mathrm{Me})_{4} \mathbf{N}\right]_{2} \mathbf{P u C l}_{6}$ \\ (Dated: July 28, 2016)
}

\section{ISOLATED PUCL ${ }_{6}{ }^{2-}$ COMPLEX}

\section{A. Electronic States in $\mathrm{PuCl}_{6}{ }^{2-}$}

The energetic splitting of the ground multiplet ${ }^{5} I_{4}$ of the $\mathrm{PuCl}_{6}{ }^{2-}$ complex were calculated at the ab-initio level with a developer's version of the Molcas software package $^{2}$ using the protocol detailed in References ${ }^{3}$ and $^{4}$. The second-order Douglas-Kroll-Hess scalar relativistic Hamiltonian ${ }^{5}$ was employed in the calculations without SO coupling. All electron ANO-RCC Gaussian-type basis sets contracted to TZP quality were employed. SO coupling was treated by state interactions between the CASSCF wave functions, using the Restricted Active Space State Interaction (RASSI) program ${ }^{6}$. Two active spaces have been investigated. They correspond to a CAS $(4,7)$, with the four unpaired electrons distributed over the seven $5 f$ orbitals, and a $\operatorname{CAS}(10,10)$ which corresponds to the previous active space augmented by three doubly occupied ligand orbitals. It has to be mentioned that the inclusion of these ligand based orbitals does not affect the nature of the ground state but leads to a small energetic stabilization of the excited states. 35 spin-free quintet states have been included in the state average calculation.

The calculated energetic splitting of the ground multiplet ${ }^{5} I_{4}$ of $\mathrm{PuCl}_{6}{ }^{2-}$ is provided in Table $\mathrm{S} 1$ for the five different geometries. In the octahedral complex, the GS corresponds to the triplet state $\Gamma_{5}$. The results from the $\mathrm{CF}$ model discussed in the main article, which is derived from the ab-initio calculated state ordering, agree numerically very well with the $a b$-initio data and therefore indicate that the $\mathrm{CF}$ model is reasonable. As seen in Figure 2 (main text), the $\Gamma_{1}$ and $\Gamma_{5}$ states are close in energy for a value of $x \approx 0.5$. The $x$ obtained from the fit is very far from this value. Only unreasonably large changes of the $\mathrm{Pu}-\mathrm{Cl}$ distances leads to $\Gamma_{1}$ and $\Gamma_{3}$ being close in energy in the ab-initio calculations.

The state ordering is not altered upon the lengthening of the $\mathrm{Pu}-\mathrm{Cl}$ distances along the $\mathrm{Z}$-axis, and the energetic separation of the GS and the excited states is hardly affected by the distortion. Importantly, though, the geometrical distortion leads to a splitting of the GS triplet $\Gamma_{5}$ into a singlet and a doublet (see Figure 3 main text). For instance, a lengthening of $0.020 \AA$ of the $\mathrm{Pu}-\mathrm{Cl}$ distances leads to a GS singlet separated from the doublet by $21 \mathrm{~cm}^{-1}$. This energetic splitting is small. However, as shown below, even a small energetic splitting of the GS triplet has a strong influence on the magnetic susceptibility below $20 \mathrm{~K}$. It should be pointed out that a small splitting of the GS triplet may not necessarily arise from a slight distortion of the octahedral CF but it may instead be due to a symmetry breaking from electrostatic interactions with the surrounding of the $\mathrm{PuCl}_{6}$ units. Without a high quality single crystal structure for temperatures below $20 \mathrm{~K}$ we cannot reliably assign the reason for an energetic splitting of the GS triplet to either a distortion of the $\mathrm{PuCl}_{6}$ octahedra or their surroundings. However, the magnetic data strongly suggest that such an energetic splitting is indeed present in the system.

The energetic splitting of the ${ }^{5} I_{4}$ ground multiplet were also calculated using the three recent experimental structures of $\mathrm{PuCl}_{6}{ }^{2-}$ characterized by Wilson at 125,298 and 360 Kelvin. ${ }^{1}$ The calculated energies are given in Table $\mathrm{S} 1$ for the three different geometries named W-125K, W$298 \mathrm{~K}$ and W-360K, respectively. The structure characterized at 360 Kelvin is of symmetry $\mathrm{O}_{h}$ with $\mathrm{Pu}-\mathrm{Cl}$ distances equal to $2.577 \AA$. Therefore, the energetic splitting of the ${ }^{5} I_{4}$ multiplet is very similar to the experimental structure characterized for this work. A small energetic stabilization of the excited states is calculated using the structure from Wilson. In the structures characterized at 298 and 125 Kelvin, the $\mathrm{Pu}-\mathrm{Cl}$ distances remain equivalent for each $\mathrm{Cl}$ atom but are slightly lengthened with $\mathrm{Pu}-\mathrm{Cl}=2.579$ and $2.585 \AA$, respectively. These two structures are slightly distorted with the $\mathrm{Cl}-\mathrm{Pu}-\mathrm{Cl}$ angles deviating from the theoretical value of $90^{\circ}$ in a $\mathrm{O}_{h}$ complex by 0.5 and $1.0^{\circ}$ in $\mathrm{W}-298 \mathrm{~K}$ and $\mathrm{W}-125 \mathrm{~K}$, respectively. These geometrical distortions lead to a splitting of the $\Gamma_{5}$ state into a singlet ground state and a low-lying first excited doublet. The first excited doublet is calculated at 24 and $48 \mathrm{~cm}^{-1}$ above the GS in W-298K and $\mathrm{W}-125 \mathrm{~K}$, respectively.

\section{B. Natural Spin Orbitals for the Ground State}

Natural spin orbitals (NSOs) and the spin magnetization $m_{z}^{S}(\vec{r})$ for a GS component of the octahedral complex with maximized positive $\left\langle S_{z}\right\rangle$ are given in Figure S1. The plots here are based on the CAS $(10,10)$ SCFSO calculation which includes three $p$-type orbitals of the ligands. Likely due to a very small breaking of the degeneracy of the GS triplet $\Gamma_{5}\left(0.5 \mathrm{~cm}^{-1}\right)$ in the calculation for $\mathrm{O}_{h}$ geometry, from numerical inaccuracies, the NSOs do not show up as $5 f$ linear combinations that are symmetry adapted for $\mathrm{O}_{h}$ but instead as $\sigma, \pi, \delta, \phi$ orbitals with some ligand contributions. The spin magnetization $m_{z}^{S}(\vec{r})$ is predominantly localized on the plutonium atom, with smaller ligand contributions than, for instance, obtained in calculations for $\mathrm{NpF}_{6}$.

The spin populations of all NSOs by definition add up to $2\left\langle S_{z}\right\rangle=\int m_{z}^{S} d V$. The major contributions to 
TABLE S1. Energetic splitting $\left(\mathrm{cm}^{-1}\right)$ of the ${ }^{5} I_{4}$ ground multiplet at the CAS(10,10)SCF-SO level (gas phase calculations). $\mathrm{T} n, n=1,2,3,4$, indicate slightly distorted tetragonal $D_{4 h}$ geometries as explained in the main text, and W-XXXK indicate experimental structures by Wilson from Reference ${ }^{1}$.

\begin{tabular}{ccccccccc}
\hline & $\mathrm{O}_{h}{ }^{a}$ & $\mathrm{~W}-360 \mathrm{~K}^{b}$ & $\mathrm{~W}-298 \mathrm{~K}^{b}$ & $\mathrm{~W}-125 \mathrm{~K}^{b}$ & $\mathrm{~T} 1$ & $\mathrm{~T} 2$ & $\mathrm{~T} 3$ & $\mathrm{~T} 4$ \\
\hline$\Gamma_{5}$ & 0 & 0 & 0 & 0 & 0 & 0 & 0 & 0 \\
& 0 & 0 & 24 & 48 & 5 & 11 & 16 & 21 \\
& 0 & 0 & 24 & 48 & 5 & 11 & 16 & 21 \\
$\Gamma_{1}$ & 755 & 739 & 822 & 763 & 756 & 757 & 758 & 759 \\
$\Gamma_{4}$ & 782 & 768 & 839 & 763 & 781 & 781 & 780 & 780 \\
& 782 & 768 & 839 & 794 & 784 & 786 & 789 & 792 \\
& 782 & 768 & 839 & 794 & 784 & 786 & 789 & 792 \\
$\Gamma_{3}$ & 825 & 812 & 868 & 846 & 826 & 828 & 829 & 831 \\
& 825 & 812 & 868 & 846 & 827 & 829 & 831 & 833 \\
\hline
\end{tabular}

${ }^{a}$ Experimental structure from this work. ${ }^{b}$ Experimental structures from Reference ${ }^{1}$.

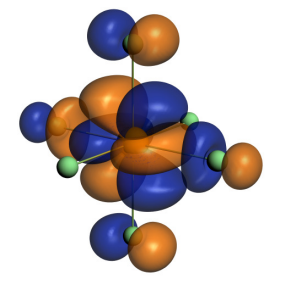

$\varphi_{\pi}^{Z}:+0.469$

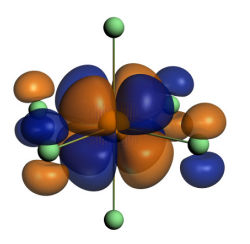

$\varphi_{\delta}^{Z}:+0.229$

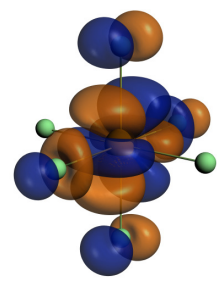

$\varphi_{\pi}^{Z}:+0.469$

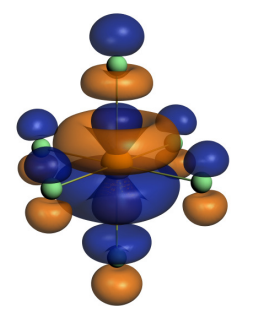

$\varphi_{\sigma}^{Z}:+0.023$

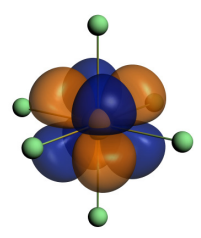

$\varphi_{\delta}^{Z}:+0.328$

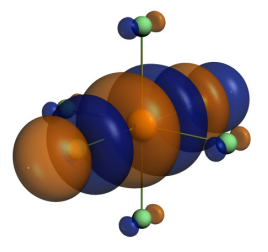

$\varphi_{p}^{Z}:+0.001$

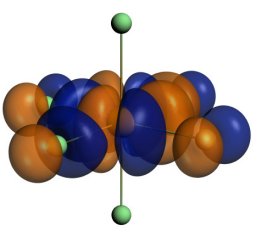

$\varphi_{\phi}^{Z}:+0.245$

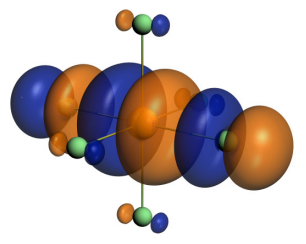

$\varphi_{p}^{Z}:+0.001$

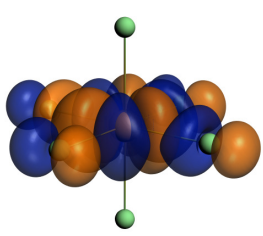

$\varphi_{\phi}^{Z}:+0.245$

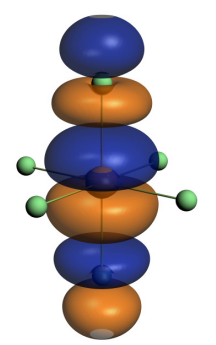

$\varphi_{p}^{Z}:+0.001$

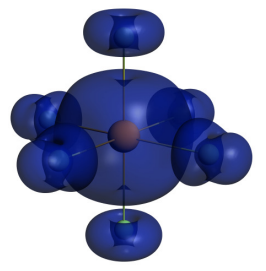

$$
\begin{gathered}
\left\langle L_{\alpha}\right\rangle=-3.379 \\
\left\langle S_{\alpha}\right\rangle=+1.007 \\
g=-1.363
\end{gathered}
$$

$$
m_{z}
$$

FIG. S1. $\mathrm{PuCl}_{6}{ }^{2-}$ : Natural spin orbitals $\varphi^{z}$ with spin- $z$ populations, and spin magnetization $m_{z}^{S}(\vec{r})$ from a CAS $(10,10) \mathrm{SCF}-\mathrm{SO}$ calculation. Isosurface values \pm 0.01 au for NSOs and \pm 0.0001 au for $m_{Z}^{S}(\vec{r})$. 

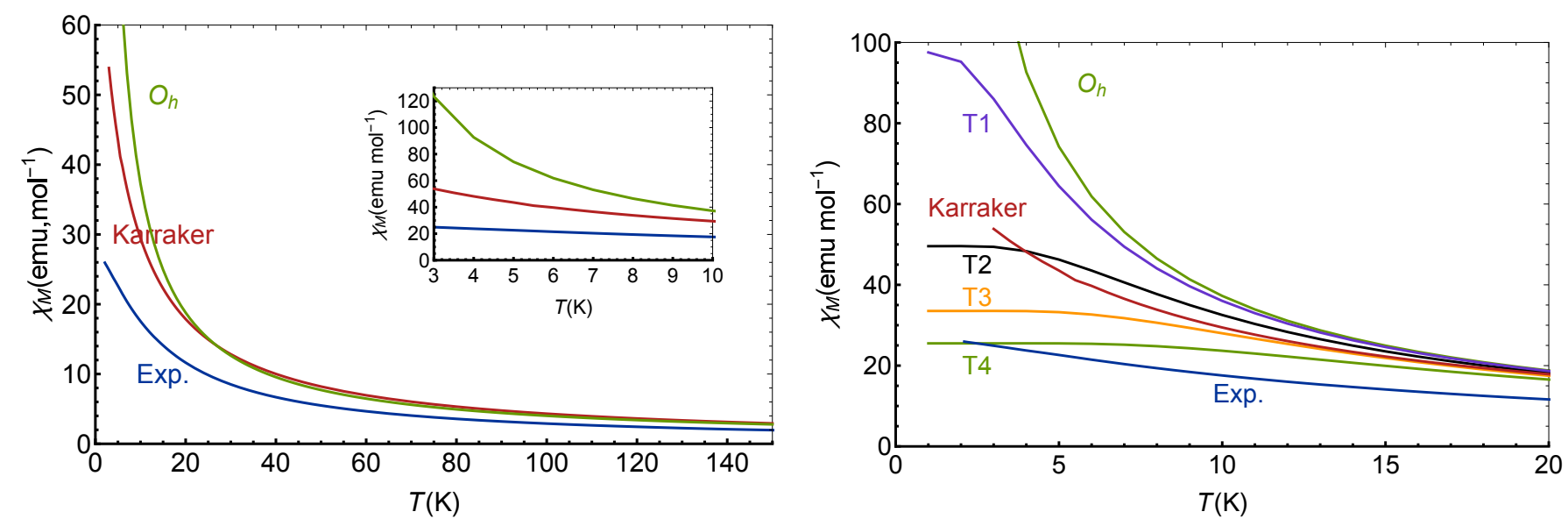

FIG. S2. $\mathrm{PuCl}_{6}{ }^{2-}$ : Calculated magnetic susceptibility $\chi\left(\mathrm{emu} \mathrm{mol}{ }^{-1} \times 10^{3}\right)$ at the CAS(10,10)SCF-SO level. Experimental data from Ref. ${ }^{7}$ and from this work.

TABLE S2. Experimental ${ }^{a}$ and calculated magnetic susceptibility $\chi\left(\mathrm{emu} \mathrm{mol}^{-1} \times 10^{3}\right)$ at selected temperatures. CAS $(10,10)$ SCF-SO results.

\begin{tabular}{lcccccccccc}
\hline T $(\mathrm{K})$ & LANL & Ref $^{7}$ & $\mathrm{O}_{h}$ & $\mathrm{~W}-360 \mathrm{~K}$ & $\mathrm{~W}-298 \mathrm{~K}$ & W125K & $\mathrm{T} 1$ & $\mathrm{~T} 2$ & $\mathrm{~T} 3$ & $\mathrm{~T} 4$ \\
\hline 3 & 24.92 & 53.84 & 123.42 & 124.27 & 26.52 & 13.15 & 86.01 & 49.40 & 33.54 & 25.51 \\
10 & 17.56 & 29.44 & 37.25 & 37.53 & 24.24 & 13.11 & 36.02 & 32.53 & 28.00 & 23.67 \\
20 & 11.63 & 17.87 & 18.79 & 18.94 & 16.75 & 12.09 & 18.65 & 18.20 & 17.48 & 16.55 \\
150 & 1.96 & 2.93 & 2.78 & 2.83 & 2.80 & 2.81 & 2.78 & 2.78 & 2.78 & 2.79
\end{tabular}

a: The experimental data from Ref. ${ }^{7}$ are extracted from the Curie-Weiss law $\chi=c /(T+\theta)$, where $C=0.455 \mathrm{emu} \mathrm{mol}^{-1}$ and $\theta=5.45 \mathrm{~K}$.
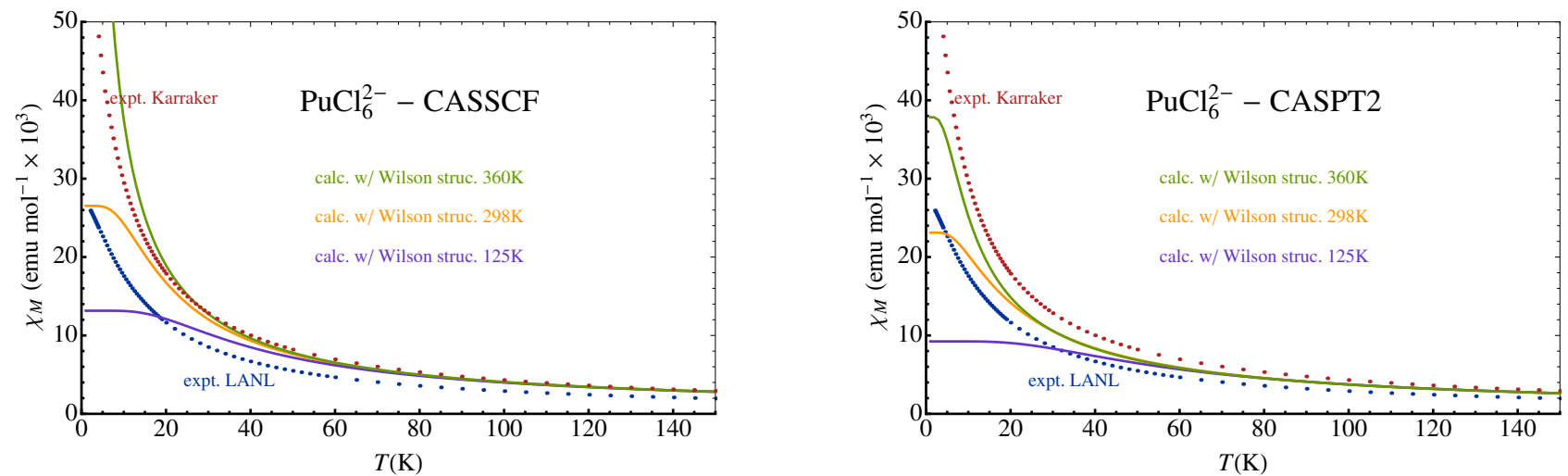

FIG. S3. $\mathrm{PuCl}_{6}{ }^{2-}$ : Calculated magnetic susceptibility $\chi\left(\mathrm{emu} \mathrm{mol}^{-1} \times 10^{3}\right)$ at the CAS(10,10)SCF-SO level $($ left $)$ and CAS(10,10)PT2-SO level (right). Experimental data from Ref. ${ }^{7}$ and from this work.

\section{Magnetic Susceptibility of $\mathrm{PuCl}_{6}{ }^{2-}$}

The calculated curves of the magnetic susceptibility $\chi$ as a function of the temperature $T$ are shown in Figure $\mathrm{S} 2$ for the different geometries and compared to the experimental data extracted from Karraker's paper and to the more recent measurements performed at Los Alamos National Lab (LANL, this work). Numerical values of $\chi$ for selected temperatures are given in Table S2.

The calculated $\chi$ for the octahedral geometry repro- duces qualitatively the experimental data. Large values of $\chi$ are calculated at very low temperatures and $\chi$ strongly decreases with the increase of $T$. At very low temperatures, the calculation overestimates both the Karraker and LANL data. For instance, at $3 \mathrm{~K}$ the calculation gives $\chi=123 \mathrm{emu} \mathrm{mol}^{-1} \times 10^{3}$, whereas experimentally $\chi=24$ and $53 \mathrm{emu} \mathrm{mol}^{-1} \times 10^{3}$ for LANL and Karraker, respectively. However, the discrepancy between theory and experiment quickly decreases for $T>10 \mathrm{~K}$ (see Table S2). At $150 \mathrm{~K}$ there is excellent agreement of the calculated $\chi$ with the susceptibility 
data of Karraker. The source of the discrepancy between the two sets of experimental data is unclear.

The differences between calculated and experimental $\chi$ values at very low temperatures can be rationalized by a small energetic splitting of the GS triplet $\Gamma_{5}$. As already mentioned, for the numerical simulations we induced such a splitting by a small tetragonal distortion of the $\mathrm{PuCl}_{6}{ }^{2-}$ complex ion but the actual source of the splitting may or may not be the same.

In the paper of Karraker, two possibilities were considered in order to rationalize the magnetic behavior at low $T$. The first one rationalized the low-temperature behavior of $\chi$ by assuming that the $\Gamma_{5}$ triplet was a minuscule $1.5-2.5 \mathrm{~cm}^{-1}$ above a $\Gamma_{1}$ ground state. This assignment is strongly contradicted by the ab-initio calculations (Table $\mathrm{S} 1)$. It is difficult to imagine distortions, different $\mathrm{Pu}-\mathrm{Cl}$ distances than those determined by the diffraction studies, or crystal packing effects, that would eliminate the greater than 700 wavenumber energy gap calculated between $\Gamma_{5}$ and $\Gamma_{1}$.

The other possibility suggested by Karraker was that the octahedral ion could potentially be sufficiently distorted to split a $\Gamma_{5}$ GS into a singlet and a doublet. A singlet only $1.5-2.5 \mathrm{~cm}^{-1}$ below a doublet, resulting from such a distortion, could account for the low- $T$ behavior of the magnetic susceptibility. In order to investigate this hypothesis, the susceptibility was calculated for the various tetragonally distorted $\mathrm{PuCl}_{6}{ }^{2-}$ complex ions mentioned above. The corresponding curves for $\chi$ are shown in Figure S2. The geometrical distortion strongly decreases $\chi$ at very low temperatures. For instance, the calculated value of $\chi$ at 3 Kelvins is $123 \mathrm{emu} \mathrm{mol}^{-1} \times 10^{3}$ for the octahedral complex but it decreases to $25 \mathrm{emu}$ $\mathrm{mol}^{-1} \times 10^{3}$ for a distortion by $0.020 \AA$, as a direct consequence of the GS triplet $\Gamma_{5}$ being split energetically. As seen in Table S1, the largest geometrical distortion leads to a singlet $21 \mathrm{~cm}^{-1}$ below a doublet. A smaller distortion resulting in a smaller energetic splitting would give very close agreement with Karraker's susceptibility. An energetic splitting of the GS triplet $\Gamma_{5}$ leads to temperature-independent paramagnetism (TIP) at very low temperatures. Above 15 Kelvin, the geometrical distortion only has a small influence on $\chi$.

The magnetic susceptibly was also calculated using the structures characterized by Wilson and the results are compared to the experimental data in Figure S3. Due to the strictly $O_{h}$ symmetry, the calculated $\chi$ in $\mathrm{W}-360 \mathrm{~K}$ strongly overestimates the experimental data with $\chi=$ 124 emu mol ${ }^{-1} \times 10^{3}$ at $3 \mathrm{~K}$. The use of the structures characterized at 298 and 125 Kelvin leads to a strong decrease of the calculated susceptibility with $\chi=26$ and 13 emu $\mathrm{mol}^{-1} \times 10^{3}$ at $3 \mathrm{~K}$, respectively. susceptibility calculated at the CASPT2 level, i.e. including dynamic correlation, is decreased. However, this decreases of $\chi$ is due to a strong symmetry breaking in the wave functions and therefore the results are not reliable.
TABLE S3. Hyperfine coupling constant $(\mathrm{MHz})$ for ${ }^{239} \mathrm{Pu}$ in $\mathrm{PuCl}_{6}{ }^{2-}$. A nuclear $g$-factor $g_{\mathrm{Pu}}=0.3^{8}$ was employed to convert the HFCs from atomic units to $\mathrm{MHz}$.

\begin{tabular}{llcccc}
\hline Active Space & State & $A$ & $A_{\mathrm{FC}}$ & $A_{\mathrm{SD}}$ & $A_{\mathrm{PSO}}$ \\
\hline $\operatorname{CAS}(10,10)$ & $\Gamma_{5}$ & 756 & 0 & 24 & 732 \\
$\operatorname{RAS}[5,20]$ & $\Gamma_{5}$ & 1360 & 592 & 24 & 743 \\
$\operatorname{RAS}[5,32]$ & $\Gamma_{5}$ & 1361 & 592 & 24 & 744 \\
\hline
\end{tabular}

\section{Hyperfine Coupling Calculations}

Calculated hyperfine coupling constant for the $\mathrm{Pu}$ center in $\mathrm{PuCl}_{6}{ }^{2-}$ are provided in Table S3. At the CAS(10,10)SCF-SO level, the calculated hyperfine coupling constant for $\mathrm{Pu}$ is $756 \mathrm{MHz}$. This value arises mainly from the paramagnetic spin-orbital (PSO) contribution $(732 \mathrm{MHz})$ and in a lesser extent from the spindipole (SD) contribution $(24 \mathrm{MHz})$. Due to the lack of spin-polarization on the metal center, the Fermi-contact (FC) contribution vanishes at this level of theory. In order to introduce some spin-polarization, the hyperfine coupling constant has been calculated at the RASSCF level of theory. Based on a CASSCF wave function, this approach introduces spin-polarization by allowing additional single excitations between occupied (RAS1) and unoccupied orbitals (RAS3) outside of the principal active space (RAS2), as well as single excitations between RAS1/3 and RAS2, without further optimization of the orbitals. Details of the procedure are provided in Reference $^{9}$.

In Table S3, two RAS spaces, namely RAS[5,20] and RAS $[5,32]$ (the two numbers indicate the size of RAS1 and RAS3, respectively), have been investigated in conjunction with a $(4,7)$ RAS2 obtained from a CAS $(4,7)$ calculation. The five doubly occupied RAS1 orbitals correspond to linear combinations of $\mathrm{Cl}$ centered orbitals with $3 s$ character with $\mathrm{Pu}$-centered orbitals. The RAS3 spaces correspond to the first 20 and first 32 unoccupied orbitals, respectively. Introduction of the spin-polarization leads to an increase of the calculated isotropic hyperfine coupling constant. Indeed, calculations give $A=$ 1360 and $1361 \mathrm{MHz}$ for $\operatorname{RAS}(5,20)$ and $\operatorname{RAS}(5,32)$, respectively. The main difference is found with the FC contribution which strongly increases with the RAS calculations. Indeed, calculations give $A^{\mathrm{FC}}=592 \mathrm{MHz}$

TABLE S4. Hyperfine coupling constant $(\mathrm{MHz})$ for ${ }^{35} \mathrm{Cl}$ in $\mathrm{PuCl}_{6}{ }^{2-}$. A nuclear $g$-factor $g_{\mathrm{Cl}}=0.54$ were employed to convert the HFCs from atomic units to $\mathrm{MHz}$.

\begin{tabular}{ccccc}
\hline & $\tilde{S}$ & $A_{\|}$ & $A_{\perp}$ & $A_{\text {iso }}$ \\
\hline Wilson 125 K & $1 / 2$ & 9.619 & 0.000 & 3.209 \\
& 1 & 2.032 & 5.872 & 4.592 \\
Wilson 298 K & $1 / 2$ & 9.816 & 0.000 & 3.272 \\
& 1 & 2.053 & 5.914 & 4.627 \\
Wilson 360 K & 1 & 2.061 & 5.930 & 4.641 \\
\hline
\end{tabular}


for both $\operatorname{RAS}(5,20)$ and $\operatorname{RAS}(5,32)$. Due to the use of nonrelativistic hyperfine integrals, the present data may somewhat overestimate the FC contribution. The chosen RAS1 space is qualitatively comparable to one chosen for $\mathrm{NpF}_{6}$ in Reference ${ }^{9}$, which gave very good agreement with experimental data for the Np hyperfine coupling. A numerical breakdown was only evident for active spaces including larger numbers of metal-centered orbitals. (An extension of the code to allow the use of relativistic hyperfine integrals is currently under way.)

Calculated hyperfine coupling constant for the $\mathrm{Cl}$ atoms in $\mathrm{PuCl}_{6}{ }^{2-}$ are provided in Table S4. The calculations were carried out using the three structures characterized by Wilson with a RAS space RAS $[6,65]$ in conjunction with a $(4,7)$ RAS2 obtained from a CAS $(4,7)$ calculation. The six doubly occupied RAS1 orbitals correspond to linear combinations of $\mathrm{Cl}$ centered orbitals with $3 s$ character. The RAS3 spaces correspond to the first 65 unoccupied orbitals. In the case of the structures characterized at 125 and 298 Kelvin, the calculations were performed using a pseudo-spin $\tilde{S}=1 / 2$ and $\tilde{S}=1$ in order to check the influence of the splitting of the $\Gamma_{5}$ GS. In the case where the GS was considered as a triplet, i.e. $\tilde{S}=1$, the isotropic hyperfine coupling constant $A_{\text {iso }}$ remains relatively constant for the three different structures with $A_{\text {iso }}=4.6 \mathrm{MHz}$. For the triplet GS, the perpendicular component is calculated larger than the parallel one with $A_{\|}=2.0 \mathrm{MHz}$ and $A_{\perp}=5.9 \mathrm{MHz}$. A major difference arises when the splitting of the $\Gamma_{5}$ state into a singlet GS and a first excited doublet is considered in $\mathrm{W}-125 \mathrm{~K}$ and $\mathrm{W}-298 \mathrm{~K}$. In these cases, a large increase of the parallel component is calculated. However, due to a perpendicular component equal to zero, the isotropic value is calculated smaller $\left(A_{\text {iso }}=3.2 \mathrm{MHz}\right)$ compared to the triplet case.

\section{E. CASSCF calculations of the chlorine EFG}

EFG tensors have further been calculated at the CAS $(10,10)$ SCF-SO level. Results for the GS triplet $\Gamma_{5}$ and the singlet excited state $\Gamma_{1}$ are provided in Table S5. An important finding is that the magnitude of the EFG does not strongly depend on the electronic configuration at the metal center.

Some further tests were performed as follows: 'Partial' AMFI integrals ${ }^{3}$ have been used to disable contributions to the $\mathrm{SO}$ coupling from the chloride atoms. As expected, the EFG are not affected by SO coupling originating from the $\mathrm{Cl}$ centers. Furthermore, the use of a contracted versus an un-contracted basis set for the chlorine atoms, i.e ANO-RCC and ANO-RCC-UnContracted, respectively, leads to a decrease of the magnitude of the EFGs. On the other hand, the use of the contracted Iglo-III basis set (designed for NMR calculations) instead of ANORCC leads to an increase of the magnitude of the EFGs. We show these data to demonstrate the variability of the EFGs when using different good quality AO basis sets.
TABLE S5. Chlorine EFG tensor components (atomic units) for $\mathrm{PuCl}_{6}{ }^{2-}$, from CAS(10,10)SCF-SO calculations.

\begin{tabular}{llllll}
\hline Cl Basis Set & & State & $V_{11}$ & $V_{22}$ & $V_{33}$ \\
\hline ANO-RCC & AMFI & $\Gamma_{5}{ }^{a}$ & -0.177 & -0.181 & 0.359 \\
& & $\Gamma_{1}$ & -0.179 & -0.179 & 0.358 \\
ANO-RCC & PAMFI & $\Gamma_{5}{ }^{a}$ & -0.177 & -0.181 & 0.358 \\
& $\Gamma_{1}$ & -0.175 & -0.175 & 0.351 \\
ANO-RCC-UnContracted AMFI & $\Gamma_{5}{ }^{a}$ & -0.155 & -0.158 & 0.313 \\
& $\Gamma_{1}$ & -0.156 & -0.156 & 0.312 \\
ANO-RCC-UnContracted PAMFI & $\Gamma_{5}{ }^{a}$ & -0.155 & -0.158 & 0.313 \\
& & $\Gamma_{1}$ & -0.156 & -0.156 & 0.312 \\
Iglo-III & AMFI & $\Gamma_{5}{ }^{a}$ & -0.193 & -0.196 & 0.389 \\
& & $\Gamma_{1}$ & -0.192 & -0.195 & 0.387 \\
Iglo-III & PAMFI & $\Gamma_{5}{ }^{a}$ & -0.193 & -0.196 & 0.389 \\
& & $\Gamma_{1}$ & -0.192 & -0.195 & 0.387 \\
\hline
\end{tabular}

$a$ : EFG tensor components averaged over the three components of the triplet.

Since the CAS calculations do not include dynamic correlation to an appreciable extent, they are best compared with the HF data of Table S8. The CAS results are bracketed by the SR data for the two available HF configurations and agree very well with the quasi-restricted HF-SO EFG tensor components. This provides us with an opportunity to extrapolate how the CAS results would change upon inclusion of dynamic correlation. The most likely outcome would be an increase similar to the one when going from HF-SO to B3LYP or PBE0 in Table S8. In the following Section we estimate effects from the surrounding of $\mathrm{PuCl}_{6}{ }^{2-}$ in the solid, which is shown to decrease the magnitude of the $\mathrm{Cl}$ EFG tensor components. Therefore, for this system, neglecting dynamic correlation as well as the embedding in the solid provides a relatively well balanced cancellation of errors.

The EFG tensors were also calculated for the structures characterized by Wilson; the results are collected in Table S6. The use of the Wilson structures leads to an increase of the magnitude of the Cl EFG tensor components compared to the results obtained with the LANL

TABLE S6. Chlorine EFG tensor components (atomic units) for $\mathrm{PuCl}_{6}{ }^{2-}$, from CAS(10,10)SCF-SO calculations. Iglo-III basis set.

\begin{tabular}{llccc}
\hline & State & $V_{11}$ & $V_{22}$ & $V_{33}$ \\
\hline LANL & $\Gamma_{5}$ & -0.193 & -0.196 & 0.389 \\
& $\Gamma_{1}$ & -0.192 & -0.195 & 0.387 \\
Wilson 125 K & $\Gamma_{5}$ & -0.208 & -0.222 & 0.431 \\
& $\Gamma_{1}$ & -0.210 & -0.217 & 0.427 \\
Wilson 298 K & $\Gamma_{5}$ & -0.205 & -0.212 & 0.417 \\
& $\Gamma_{1}$ & -0.206 & -0.207 & 0.414 \\
Wilson 360 K & $\Gamma_{5}$ & -0.205 & -0.207 & 0.412 \\
& $\Gamma_{1}$ & -0.202 & -0.206 & 0.408 \\
\hline
\end{tabular}

${ }^{a}$ : EFG tensor components averaged over the three components of the triplet. 


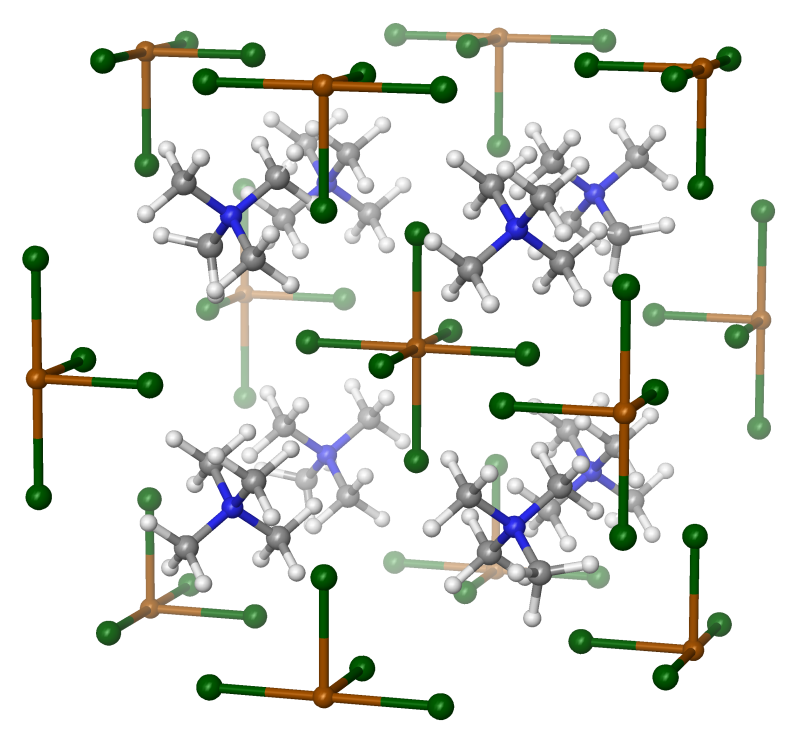

FIG. S4. $\left[\left(\mathrm{CH}_{3}\right)_{4} \mathrm{~N}\right]_{2} \mathrm{PuCl}_{6} \cdot \mathrm{PuCl}_{6}{ }^{2-}$ surrounded by a first shell of counter ions and nearby $\mathrm{Pu}$ and $\mathrm{Cl}$ centers.

structure.

\section{F. DFT and Hartree-Fock calculations of the chlorine EFG}

EFG calculations have been performed on the experimental structure Hartree-Fock theory and DFT with the ADF package. ${ }^{10-12}$ The results are collected in Table S8. Scalar relativistic (SR) calculations were carried out for a spin quintuplet. In octahedral symmetry, the $5 f$ orbitals are split into one $a_{2 u}$ and one set each of $t_{2 u}$ and $t_{1 u}$. Three different high-spin electronic configurations were considered at the SR level: $a_{2 u}^{1} t_{2 u}^{3} t_{1 u}^{0}, a_{2 u}^{1} t_{2 u}^{2} t_{1 u}^{1}$ and $a_{2 u}^{1} t_{2 u}^{0} t_{1 u}^{3}$. Other possible spin states, i.e. triplets and singlets, were not not considered because they were found at much higher energies (the first triplet and singlet were calculated at 14160 and $22480 \mathrm{~cm}^{-1}$ above the quintuplet). The EFGs were also calculated with DFT and HF including SO coupling, using two different types of calculations: (i) quasi-restricted (no spin polarization) and (ii) unrestricted (spin-polarized). The meaning of the number of 'unpaired spins' in the latter calculations is not well defined.

The following trends can be noticed in Table S8: (i) In the SR calculations, the occupation scheme $a_{2 u}^{1} t_{2 u}^{3} t_{1 u}^{0}$ always gives the largest values for the EFG tensor components, irrespective of the functional. The magnitude of the EFG decreases when the $t_{2 u}$ set is depopulated in favor of the $t_{1 u}$ set. For instance with the PBE functional, $V_{11}=V_{22}=-0.32 \mathrm{au}$ and $V_{33}=0.64$ au for the occupation scheme $a_{2 u}^{1} t_{2 u}^{3} t_{1 u}^{0}$, whereas $V_{11}=V_{22}=-0.05$ au and $V_{33}=0.11$ au in the case of $a_{2 u}^{1} t_{2 u}^{0} t_{1 u}^{3}$. (ii) With increasing amount of exact exchange in the functional, going from PBE to HF, the magnitude of the EFG tensor components decreases. This trend is also found for the quasi-restricted SO calculations, with the latter producing EFGs that are closest to the SR $a_{2 u}^{1} t_{2 u}^{2} t_{1 u}^{1}$ data (the pure HF calculation did not converge properly and the results are omitted from the table). The spin-polarized SO calculations give less systematic results. (iii) With increasing amount of exact exchange in the functional, the differences of the EFGs between the $a_{2 u}^{1} t_{2 u}^{3} t_{1 u}^{0}$ and the $a_{2 u}^{1} t_{2 u}^{0} t_{1 u}^{3}$ configurations decrease.

\section{EMBEDDED PUCL ${ }_{6}{ }^{2-}:\left[\left(\mathrm{CH}_{3}\right)_{4} \mathrm{~N}\right]_{2} \mathrm{PUCL}_{6}$}

The influence of the environment on the electronic structure and the magnetic properties of the $\mathrm{PuCl}_{6}{ }^{2-}$ anion have been investigated with DFT and ab-initio embedding calculations, Calculations for an embedded $\left[\left(\mathrm{CH}_{3}\right)_{4} \mathrm{~N}\right]_{2} \mathrm{PuCl}{ }_{6}$ complex ion have been performed based on the experimental structure determined in this work. Prior to the embedding calculations, the hydrogen positions of the TMA units in the solid were optimized with periodic boundary conditions.

The geometry optimizations were performed with DFT, as implemented in the Vienna ab-initio Simulation Package (VASP) version 5.3.2. ${ }^{13}$ The projector augmented wave (PAW) method was used to treat the core states. ${ }^{14}$ For the optimizations the $\mathrm{Pu} 5 f / 6 d / 6 p / 7 s$, N $2 s / 2 p, \mathrm{C} 2 s / 2 p, \mathrm{Cl} 3 s / 3 p$ and $\mathrm{H} 1 s$ electrons were treated explicitly, and the standard PAW potentials were employed, along with an energy cutoff of $500 \mathrm{eV}$. The gradient corrected exchange and correlation functional of Perdew-Burke-Ernzerhof (PBE) was used. ${ }^{15}$ The $k$-point grids were generated using the automatic $\Gamma$-centered Monkhorst-Pack scheme, and the number of divisions along each reciprocal lattice vecor was chosen such that the product of this number with the real lattice constant was $50 \AA$. We employed DFT $+\mathrm{U}$ using the rotationally invariant method of Dudarev with a $U_{\text {eff }}$ of $3.6 \mathrm{eV}$ for the $\mathrm{Pu} f$ shells. ${ }^{16}$

The DFT and ab-initio embedding calculations then correspond to a $\mathrm{PuCl}_{6}{ }^{2-}$ ion surrounded by 1096 point charges representing the 1096 nearest atoms in the solid. For illustration, the $\mathrm{PuCl}_{6}{ }^{2-}$ unit surrounded by a first shell of counter ions and neighboring $\mathrm{Pu}$ and $\mathrm{Cl}$ centers is shown in Figure S4. The atomic charges $q$ used for the $\left[\left(\mathrm{CH}_{3}\right)_{4} \mathrm{~N}\right]_{2} \mathrm{PuCl}_{6}$ crystal were deduced from a 'Loprop' calculation at the CAS(4,7)SCF level for $\mathrm{PuCl}_{6}{ }^{2-}$ and at the $\mathrm{CAS}(6,4) \mathrm{SCF}$ level for $\left[\left(\mathrm{CH}_{3}\right)_{4} \mathrm{~N}\right]^{+}$, similar to a procedure recently used for neptunyl complex ions ${ }^{3}$.

\section{A. Electronic States with Embedding}

The influence of the environment on the energetic splitting of the ${ }^{5} I_{4}$ GS of the $\mathrm{PuCl}_{6}{ }^{2-}$ complex were investigated at the $\operatorname{CAS}(10,10) \mathrm{SCF}-\mathrm{SO}$ level. The calculated 
TABLE S7. Chlorine EFG tensor components (atomic units) for $\mathrm{PuCl}_{6}{ }^{2-}$, from CAS(10,10)SCF-SO calculations. Iglo-III basis set.

\begin{tabular}{|c|c|c|c|c|c|c|c|c|c|c|}
\hline \multirow[b]{2}{*}{ Structure } & \multirow[b]{2}{*}{ State } & \multicolumn{3}{|c|}{ Average $\mathrm{Cl}^{a}$} & \multicolumn{3}{|c|}{ Axial $\mathrm{Cl}^{b}$} & \multicolumn{3}{|c|}{ Equatorial $\mathrm{Cl}^{c}$} \\
\hline & & $V_{11}$ & $V_{22}$ & $V_{33}$ & $V_{11}$ & $V_{22}$ & $V_{33}$ & $V_{11}$ & $V_{22}$ & $V_{33}$ \\
\hline $\mathrm{O}_{h}$ & Triplet & -0.193 & -0.196 & 0.389 & -0.193 & -0.196 & 0.389 & -0.193 & -0.196 & 0.389 \\
\hline \multirow[t]{2}{*}{$\mathrm{T} 1$} & Singlet & -0.196 & -0.198 & 0.394 & -0.190 & -0.190 & 0.381 & -0.199 & -0.202 & 0.401 \\
\hline & Doublet & -0.195 & -0.199 & 0.394 & -0.202 & -0.207 & 0.410 & -0.191 & -0.195 & 0.386 \\
\hline \multirow[t]{2}{*}{$\mathrm{T} 2$} & Singlet & -0.198 & -0.199 & 0.398 & -0.195 & -0.195 & 0.391 & -0.200 & -0.201 & 0.402 \\
\hline & Doublet & -0.196 & -0.202 & -0.398 & -0.208 & -0.212 & 0.420 & -0.190 & -0.196 & 0.387 \\
\hline \multirow[t]{2}{*}{$\mathrm{T} 3$} & Singlet & -0.200 & -0.201 & 0.402 & -0.200 & -0.200 & 0.401 & -0.201 & -0.201 & 0.402 \\
\hline & Doublet & -0.197 & -0.204 & 0.401 & -0.212 & -0.217 & 0.429 & -0.190 & -0.198 & 0.387 \\
\hline \multirow[t]{2}{*}{$\mathrm{T} 4$} & Singlet & -0.202 & -0.203 & 0.405 & -0.205 & -0.205 & 0.411 & -0.200 & -0.202 & 0.403 \\
\hline & Doublet & -0.198 & -0.206 & 0.405 & -0.217 & -0.221 & 0.438 & -0.189 & -0.199 & 0.388 \\
\hline
\end{tabular}

TABLE S8. DFT Electric Field Gradient (atomic units) obtained for the $\mathrm{Cl}$ centers of the $\mathrm{PuCl}_{6}{ }^{2-}$ ion. ADF code, TZ2P basis set.

\begin{tabular}{|c|c|c|c|c|c|c|}
\hline & & & Configuration & $V_{11}$ & $V_{22}$ & $V_{33}$ \\
\hline \multirow[t]{5}{*}{$\mathrm{HF}$} & SR & & $a_{2 u}^{1} t_{2 u}^{3} t_{1 u}^{0}$ & -0.224 & -0.224 & 0.448 \\
\hline & SR & & $a_{2 u}^{1} t_{2 u}^{2} t_{1 u}^{1}$ & - & - & - \\
\hline & SR & & $a_{2 u}^{1} t_{2 u}^{0} t_{1 u}^{3}$ & -0.157 & -0.157 & 0.315 \\
\hline & $\mathrm{SO}$ & Restricted & & -0.182 & -0.182 & 0.363 \\
\hline & $\mathrm{SO}$ & UnRestricted & & -0.154 & -0.154 & 0.309 \\
\hline \multirow[t]{5}{*}{ PBE } & SR & & $a_{2 u}^{1} t_{2 u}^{3} t_{1 u}^{0}$ & -0.319 & -0.319 & 0.638 \\
\hline & SR & & $a_{2 u}^{1} t_{2 u}^{2} t_{1 u}^{1}$ & -0.247 & -0.247 & 0.494 \\
\hline & SR & & $a_{2 u}^{1} t_{2 u}^{0} t_{1 u}^{3}$ & -0.056 & -0.056 & 0.113 \\
\hline & $\mathrm{SO}$ & Restricted & & -0.253 & -0.253 & 0.506 \\
\hline & $\mathrm{SO}$ & UnRestricted & & -0.315 & -0.314 & 0.629 \\
\hline \multirow[t]{5}{*}{ PBE0 } & SR & & $a_{2 u}^{1} t_{2 u}^{3} t_{1 u}^{0}$ & -0.313 & -0.313 & 0.627 \\
\hline & SR & & $a_{2 u}^{1} t_{2 u}^{2} t_{1 u}^{1}$ & -0.237 & -0.237 & 0.475 \\
\hline & SR & & $a_{2 u}^{1} t_{2 u}^{0} t_{1 u}^{3}$ & -0.131 & -0.131 & 0.263 \\
\hline & $\mathrm{SO}$ & Restricted & & -0.279 & -0.279 & 0.558 \\
\hline & $\mathrm{SO}$ & UnRestricted & & -0.143 & -0.143 & 0.287 \\
\hline \multirow[t]{5}{*}{ B3LYP } & SR & & $a_{2 u}^{1} t_{2 u}^{3} t_{1 u}^{0}$ & -0.299 & -0.299 & 0.599 \\
\hline & SR & & $a_{2 u}^{1} t_{2 u}^{2} t_{1 u}^{1}$ & -0.224 & -0.225 & 0.451 \\
\hline & SR & & $a_{2 u}^{1} t_{2 u}^{0} t_{1 u}^{3}$ & -0.103 & -0.103 & 0.207 \\
\hline & $\mathrm{SO}$ & Restricted & & -0.257 & -0.257 & 0.581 \\
\hline & $\mathrm{SO}$ & UnRestricted & & -0.128 & -0.128 & 0.257 \\
\hline
\end{tabular}

TABLE S9. Atomic charges $q$ used in the DFT and ab-initio embedding calculations for the $\left[\left(\mathrm{CH}_{3}\right)_{4} \mathrm{~N}\right]_{2} \mathrm{PuCl}_{6}$ crystal.

\begin{tabular}{lc}
\hline & $q$ \\
\hline $\mathrm{Pu}$ & 3.046 \\
$\mathrm{Cl}$ & -0.841 \\
$\mathrm{~N}$ & 0.157 \\
$\mathrm{C}$ & -0.174 \\
$\mathrm{H}$ & 0.128 \\
\hline
\end{tabular}

energies are collected in Table S10.
TABLE S10. Energetic splitting $\left(\mathrm{cm}^{-1}\right)$ of the ${ }^{5} I_{4}$ ground multiplet at the CAS $(10,10)$ SCF-SO level with embedding.

\begin{tabular}{cccccc}
\hline & $\mathrm{O}_{h}$ & $\mathrm{~T} 1$ & $\mathrm{~T} 2$ & $\mathrm{~T} 3$ & $\mathrm{~T} 4$ \\
\hline$\Gamma_{5}$ & 0 & 0 & 0 & 0 & 0 \\
& 0 & 5 & 11 & 17 & 22 \\
& 0 & 5 & 11 & 17 & 22 \\
$\Gamma_{1}$ & 773 & 774 & 775 & 776 & 777 \\
$\Gamma_{4}$ & 799 & 799 & 799 & 798 & 798 \\
& 799 & 801 & 804 & 807 & 809 \\
& 799 & 801 & 804 & 807 & 809 \\
$\Gamma_{3}$ & 843 & 845 & 847 & 848 & 850 \\
& 843 & 845 & 848 & 850 & 852 \\
\hline
\end{tabular}

Similar to the gas phase calculations, the GS of the embedded octahedral $\mathrm{PuCl}_{6}{ }^{2-}$ complex corresponds also to the triplet state $\Gamma_{5}$. The first excited state is the singlet $\Gamma_{1}, 773 \mathrm{~cm}^{-1}$ above the GS. The second triplet $\Gamma_{4}$ and the doublet $\Gamma_{3}$ are calculated at 799 and 843 $\mathrm{cm}^{-1}$ above the GS. This means that the state ordering is unchanged, and the energies are similar to those of the free $\mathrm{PuCl}_{6}{ }^{2-}$ ion. The main influence of the embedding is a slight energetic destabilization of the excited states.

\section{B. Magnetic Susceptibility with Embedding}

The calculated magnetic susceptibilities $\chi$ for the embedded systems, $\mathrm{O}_{h}$ and distorted, are plotted in Figure S5 and are compared to experimental data. The presence of the embedding has virtually no impact on the magnetic susceptibility and only leads to small decrease of $\chi$ at low $T$. 


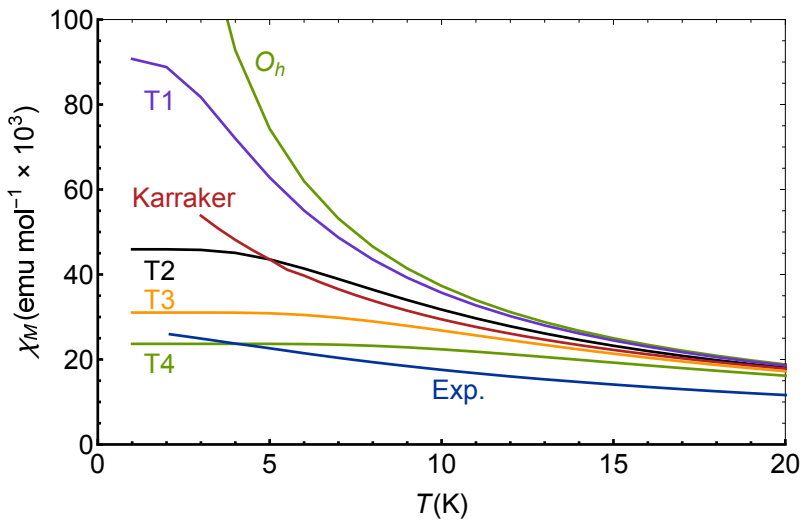

FIG. S5. $\left[\left(\mathrm{CH}_{3}\right)_{4} \mathrm{~N}\right]_{2} \mathrm{PuCl}_{6}$ : Calculated magnetic susceptibility $\chi\left(\mathrm{emu} \mathrm{mol}{ }^{-1} \times 10^{3}\right)$ at the $\operatorname{CAS}(10,10)$ SCF-SO level with embedding. Experimental data from Ref. ${ }^{7}$ and from LANL.

TABLE S11. Ab-inito Electric Field Gradient (atomic units) at the $\mathrm{Cl}$ centers of an $\mathrm{PuCl}_{6}{ }^{2-}$ ion with electrostatic embedding.

\begin{tabular}{lcccc}
\hline Cl Basis Set & State & $V_{11}$ & $V_{22}$ & $V_{33}$ \\
\hline ANO-RCC & AMFI $\Gamma_{5}{ }^{a}$ & -0.116 & -0.118 & 0.235 \\
& $\Gamma_{1}$ & -0.116 & -0.116 & 0.234 \\
\hline
\end{tabular}

${ }^{a}$ : EFG tensor components averaged over the three components of the triplet.

\section{EFGs with Embedding}

The ab-initio EFGs calculated with CAS and the embedding are collected in Table S11. The magnitudes of the EFG tensor components are reduced in the presence of the environment. As already pointed out above, the inclusion of dynamic correlation in the calculation would likely increase the EFG magnitude, both in gas phase and with embedding.

The EFG tensors have been also calculated with DFT and embedding; the results are collected in Table S12. In order to avoid unwanted symmetry breaking due to the presence of the embedding, point charges located outside of a radius $r_{0}=12 \AA$ from the central ion where screened by and exponential function. Nonetheless, the local symmetry of the Cl EFG tensors is distorted compared to the free $\mathrm{PuCl}_{6}{ }^{2-}$ complex ion, in particular for the SO-DFT calculations. Similar to the CASSCF calculations, a decrease of the magnitude of the EFG tensor components is predicted by DFT when the environment is taken into account. As pointed out above, effects from correlation and environment cancel to a degree, and the SO DFT $V_{33}$ for the embedded complex ion with the hybrid functional PBE0 is indeed comparable to those from the gas-phase CAS-SO calculations.
1 R. E. Wilson, Inorganic Chemistry 54, 10208 (2015).

2 F. Aquilante, L. De Vico, N. Ferré, G. Ghigo, P. Malmqvist, P. Neogrády, T. B. Pedersen, M. Pitoňák, M. Reiher, B. O. Roos, L. Serrano-Andrés, M. Urban, V. Veryazov, and R. Lindh, J. Comput. Chem. 31, 224 (2010).

3 F. Gendron, D. Páez-Hernández, F.-P. Notter, B. Pritchard, H. Bolvin, and J. Autschbach, Chem. Eur. J. 20, 7994 (2014).

4 F. Gendron, B. Pritchard, H. Bolvin, and J. Autschbach, Inorg. Chem. 53, 8577 (2014).

5 A. Wolf, M. Reiher, and B. A. Hess, J. Chem. Phys. 117, 9215 (2002).

6 P.-A. Malmqvist, B. O. Roos, and B. Schimmelpfennig, Chem. Phys. Lett. 357, 230 (2002).

7 D. G. Karraker, Inorg. Chem. 10, 1564 (1971).

${ }^{8}$ H. Yasuoka, G. Koutroulakis, H. Chudo, S. Richmond, D. K. Veirs, A. I. Smith, E. D. Bauer, J. D. Thompson, G. D. Jarvinen, and D. L. Clark, Science 336, 901 (2012).

9 K. Sharkas, B. Pritchard, and J. Autschbach, J. Chem. Theory Comput. 11, 538 (2015).

10 G. te Velde, F. M. Bickelhaupt, E. J. Baerends, S. J. A. van Gisbergen, C. Fonseca Guerra, J. G. Snijders, and T. Ziegler, J. Comput. Chem. 22, 931 (2001).

11 C. Fonseca Guerra, J. G. Snijders, G. Te Velde, and E. J. Baerends, Theor. Chem. Acc. 99, 391 (1998).

12 E. J. Baerends, T. Ziegler, J. Autschbach, D. Bashford, A. Bérces, F. M. Bickelhaupt, C. Bo, P. M. Boerrigter, L. Cavallo, D. P. Chong, L. Deng, R. M. Dickson, D. E. Ellis, M. van Faassen, L. Fan, T. H. Fischer, C. Fon-
TABLE S12. DFT Electric Field Gradient (atomic units) at the $\mathrm{Cl}$ centers of an $\mathrm{PuCl}_{6}{ }^{2-}$ ion with electrostatic embedding. TZ2P basis set.

\begin{tabular}{llcccc}
\hline \multicolumn{3}{c}{ Configuration } & $V_{11}$ & $V_{22}$ & $V_{33}$ \\
\hline PBE & SR & $a_{2 u}^{1} t_{2 u}^{3} t_{1 u}^{0}$ & -0.244 & -0.248 & 0.492 \\
& SO Restricted & & -0.130 & -0.168 & 0.298 \\
PBE0 & SR & $a_{2 u}^{1} t_{2 u}^{3} t_{1 u}^{0}$ & -0.240 & -0.243 & 0.483 \\
& SO Restricted & & -0.156 & -0.221 & 0.377 \\
B3LYP & SR & $a_{2 u}^{1} t_{2 u}^{3} t_{1 u}^{0}$ & -0.222 & -0.226 & 0.449 \\
& SO Restricted & & -0.136 & -0.203 & 0.339 \\
\hline
\end{tabular}

seca Guerra, A. Ghysels, A. Giammona, S. J. A. van Gisbergen, A. W. Götz, J. A. Groeneveld, O. V. Gritsenko, M. Grüning, S. Gusarov, F. E. Harris, P. van den Hoek, C. R. Jacob, H. Jacobsen, L. Jensen, J. W. Kaminski, G. van Kessel, F. Kootstra, A. Kovalenko, M. V. Krykunov, E. van Lenthe, D. A. McCormack, A. Michalak, M. Mitoraj, J. Neugebauer, V. P. Nicu, L. Noodleman, V. P. Osinga, S. Patchkovskii, P. H. T. Philipsen, D. Post, C. C. Pye, W. Ravenek, J. I. Rodríguez, P. Ros, P. R. T. Schipper, G. Schreckenbach, J. S. Seldenthuis, M. Seth, J. G. Snijders, M. Solà, M. Swart, D. Swerhone, G. te Velde, P. Vernooijs, L. Versluis, L. Visscher, O. Visser, F. Wang, T. A. Wesolowski, E. M. van Wezenbeek, G. Wiesenekker, S. K. Wolff, T. K. Woo, and A. L. Yakovlev, "Amsterdam density functional, scm, Theoreti- 
cal Chemistry, Vrije Universiteit, Amsterdam, The Netherlands." URL http://www.scm. com. Accessed 05/15.

13 G. Kresse and J. Hafner, Phys. Rev. B 47, R558 (1993).

14 P. E. Blöchl, Phys. Rev. B 50, 17953 (1994).

15 J. P. Perdew, K. Burke, and M. Ernzerhof, Phys. Rev. Lett. 77, 3865 (1996).

16 S. L. Dudarev, G. A. Botton, S. Y. Savrasov, C. J. Humphreys, and A. P. Sutton, Phys. Rev. B 57, 1505 (1998).
TABLE S13. VASP-DFT Electric Field Gradient (atomic units) at the $\mathrm{Cl}$ centers.

\begin{tabular}{lcccc}
\hline $\mathrm{PAW}^{a}$ & ENCUT & $V_{11}$ & $V_{22}$ & $V_{33}$ \\
\hline \multicolumn{4}{l}{ Fully } & Relaxed \\
$\mathrm{Pu}$ & 500 & -0.266 & -0.266 & 0532 \\
$\mathrm{Pu}$ & 600 & -0.266 & -0.266 & 0.532 \\
$\mathrm{Pu}_{s}$ & 500 & -0.267 & -0.267 & 0.534 \\
$\mathrm{H}-\mathrm{Only}$ & Relaxed & Geometry & \\
$\mathrm{Pu}$ & 500 & -0.224 & -0.224 & 0.449 \\
$\mathrm{Pu}$ & 600 & -0.224 & -0.224 & 0.449 \\
$\mathrm{Pu}_{s}$ & 500 & -0.227 & -0.227 & 0.454 \\
\hline
\end{tabular}

a $\mathrm{Pu}$ denotes a "hard" PAW with a cutoff radius of $2.20 \AA$, whereas $\mathrm{Pu}_{s}$ denotes a "soft" PAW with a cutoff radius of $3.10 \AA$ A. 